\title{
An optically directed HI search for new dwarf members of the M 81 group
}

\author{
W.K. Huchtmeier ${ }^{1}$ and E.D. Skillman ${ }^{2}$ \\ 1 Max-Planck-Institut für Radioastronomie, Auf dem Hügel 69, D-53121 Bonn, Germany \\ ${ }^{2}$ University of Minnesota, Astronomy Department, 116 Church St., SE, Minneapolis, MN 55455, U.S.A.
}

Received March 13; accepted May 9, 1997

\begin{abstract}
We present a search for neutral hydrogen (HI) emission from optically identified dwarf galaxy candidates in the larger M 81 group area. Of 136 candidates, 68 galaxies have been detected (this corresponds to a detection rate of $\sim 49 \%$ ). Most of the detected galaxies are background objects and fail to be classified as dwarfs. Many known dwarfs in the M 81 group have been detected, but no new members have been discovered.
\end{abstract}

Key words: galaxies: irregular — radio lines: galaxies - galaxies: M 81 - galaxies clusters M 81 group

\section{Introduction}

\subsection{Searching for dwarf galaxies}

Until very recently, the Las Campanas surveys of the Virgo cluster (Binggeli et al. 1985) and the Fornax cluster (Ferguson 1989) were about the only galaxy catalogs complete down to low luminosities. The Local Group and the catalog of nearby galaxies (Kraan-Korteweg \& Tammann 1979; KKT) are easily affected by incompleteness (e.g., Irwin et al. 1990), especially in the zone of avoidance (e.g., Kraan-Korteweg et al. 1994; Huchtmeier et al. 1995; McCall \& Buta 1995; Huchtmeier et al. 1997). Studies of nearby groups of galaxies were usually confined to only a small part of the sky and, due to the groups' small distances, are not sensitivity limited.

Recently, Côté (1995) has surveyed the nearby Sculptor and Centaurus groups, and discovered nine new dwarf members. This adds support to the notion that the census of nearby galaxies, particularly low surface brightness galaxies, is far from complete (e.g., Shade \& Ferguson 1994).

There are several good reasons to look for more nearby dwarf galaxies. The first, obvious reason is to improve

Send offprint requests to: W.K. Huchtmeier completeness of the sample of nearby galaxies (e.g., KKT) for the luminosity function of the "field sample".

A second goal is a study of group dynamics. HI spectra provide the only practical method for obtaining radial velocities of the very low surface brightness galaxies. A large population of dwarf galaxies with known radial velocities will allow a new estimate of the mass distribution of the M 81 group. The finding of Bothun et al. (1987) that a previously cataloged dwarf galaxy in the Virgo cluster is indeed a massive low surface brightness background galaxy stresses both the need for radial velocity measurements in such studies and the opportunity for important serendipitous discoveries.

A further motivation is to find additional very low mass galaxies. Without velocity information it is impossible to distinguish between truly low mass galaxies and low surface brightness background galaxies. HI line-widths give an additional clue to discriminate against higher mass systems. Skillman et al. $(1988,1989 a, b)$ have shown that extremely low mass galaxies hold the most promise for finding regions of near-primordial abundances. Follow-up CCD H-alpha imaging of the most promising candidates can then lead to the identification of HII regions for chemical abundance studies. The lowest mass galaxies are also important for helping to determine the trend of mass-tolight ratio with total luminosity (Côté 1995).

\subsection{The M 81 Group}

In his review of nearby groups of galaxies, de Vaucouleurs (1975) identified the M 81 group as the second closest group to ours at a distance of 2.5 Mpc. Modern Cepheid distance measurements of M 81 place it at a distance of 3.6 Mpc (Madore et al. 1993). de Vaucouleurs noted that the precise extent of the M 81 group was not well determined, as it appeared that other nearby galaxies (e.g., NGC 2403, NGC 2366, NGC 4236) could be outlying members of the M 81 group, which, itself, might be a condensation in a larger "cloud". Geller \& Huchra (1983) identified the M 81 group 

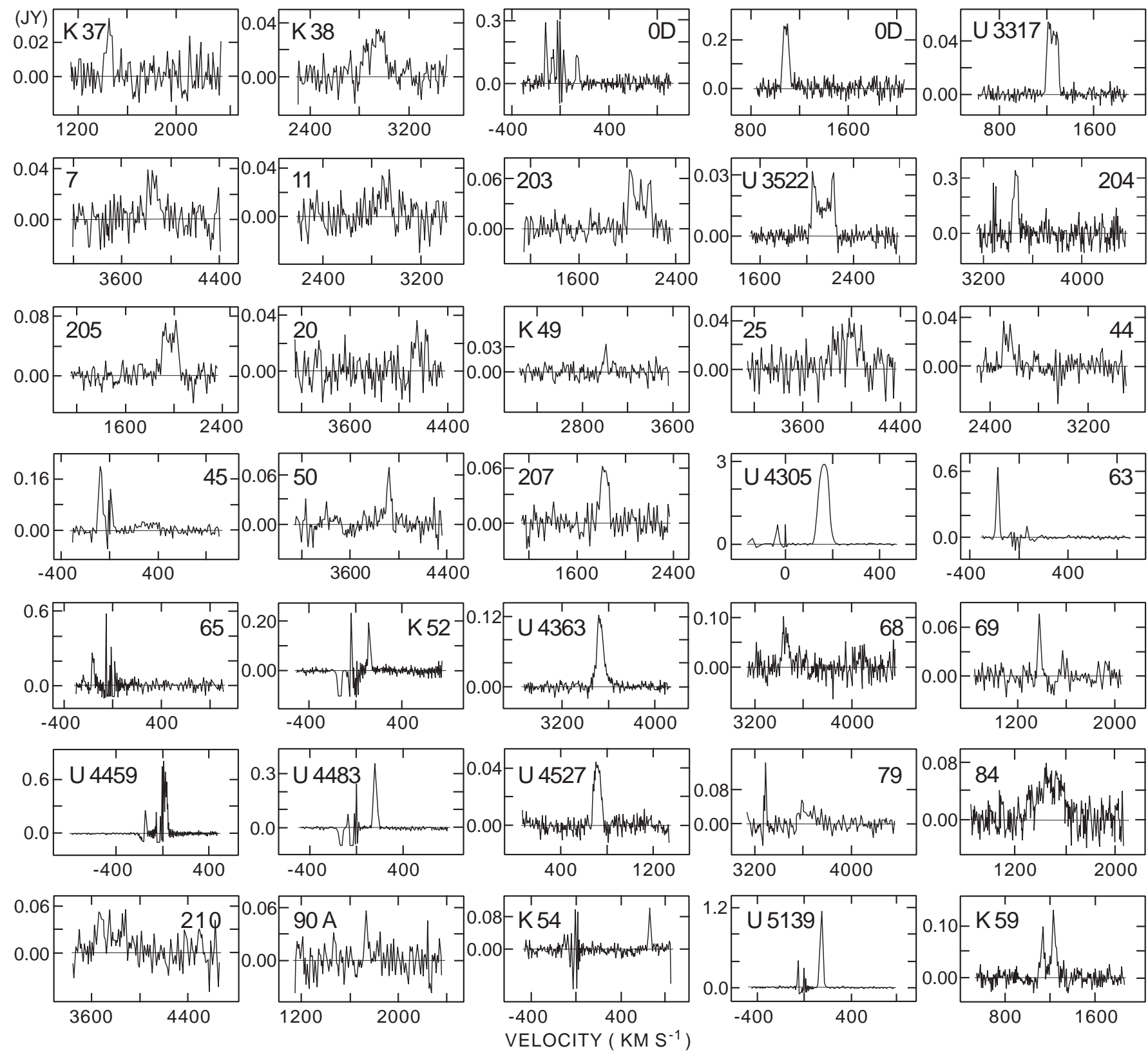

Fig. 1. a) H I profiles of the detected galaxies as seen with the $100-\mathrm{m}$ radio telescope at Effelsberg which has a half power beam width of $9.3^{\prime}$ at $21-\mathrm{cm}$ wavelength. As local $\mathrm{HI}$ is observed all over the sky our ( $\mathrm{ON}-\mathrm{OFF}$ ) observing procedure produces the difference of the local emission between the source and the reference position at local velocities around zero $\mathrm{km} \mathrm{s}^{-1}$

(their group No. 52) among the bright galaxies contained in the CfA catalog as a smaller, more concentrated distribution than de Vaucouleurs.

Tully (1988) produced a catalog of galaxies judged to be reasonably complete out to $\sim 25 \mathrm{Mpc}$. Using this catalog, Tully (1987) identified groups (thought to be gravitationally bound and virialized entities), associations, and clouds. From his analysis, the M 81 group is found to be more extended (in agreement with de Vaucouleurs original definition) and lying in the Coma - Sculptor Cloud, which contains many familiar nearby groups (M 81, the Local Group, the Sculptor Group, and the Centaurus Group).

Given the extended nature of the M 81 group, it was clear that a successful search would need to cover a large area of the sky. There are several catalogs containing optical information on M 81 dwarf galaxies; we have used the catalogs by Karachentseva (1968), Börngen et al. \& Karachentseva (1982) and Börngen et al. (1982) who list 39 faint probable members of the M 81 group excluding the known bright galaxies that usually are taken to identify this group (e.g., de Vaucouleurs 1975). These dwarf 

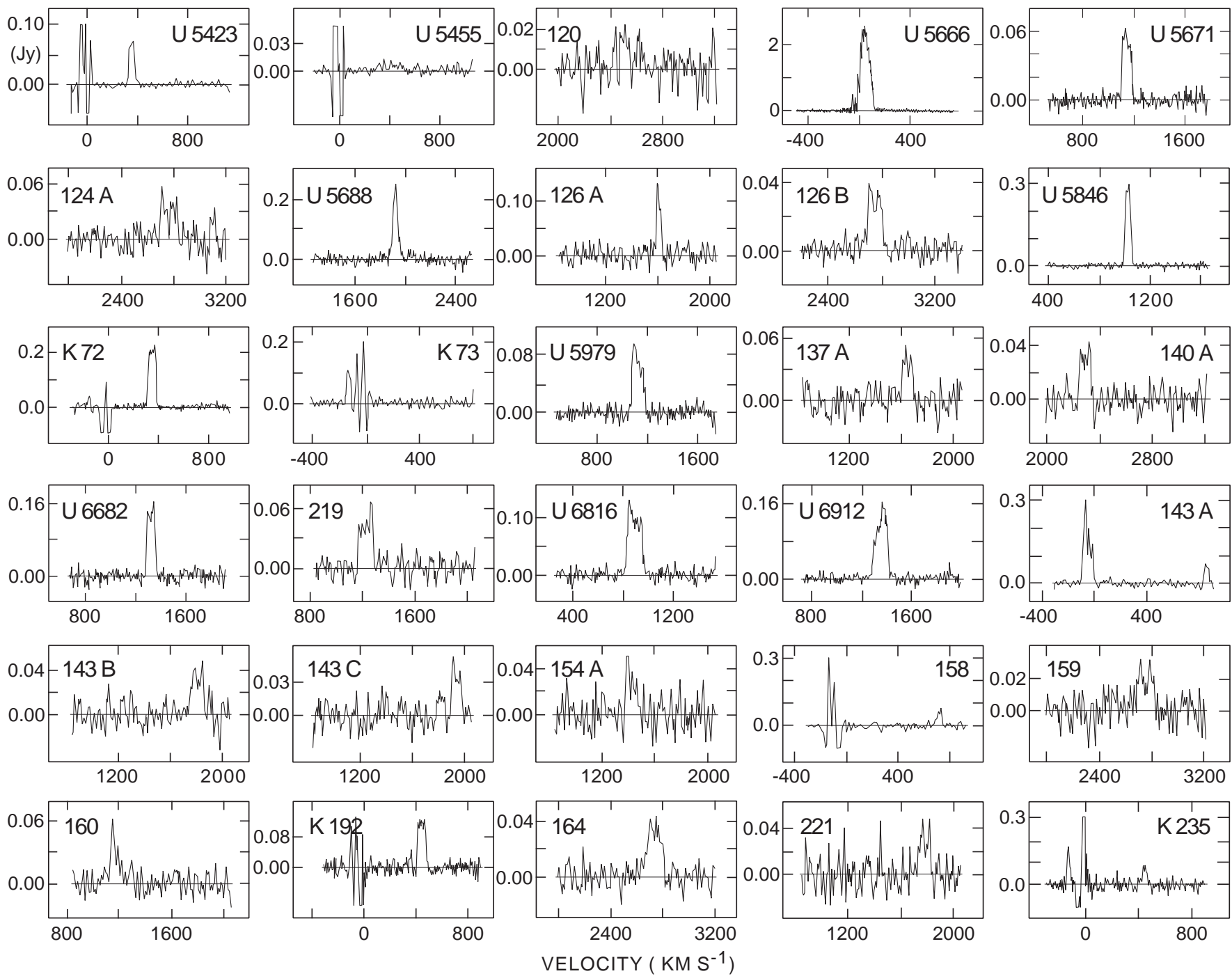

Fig. 1. b) H I profiles of the detected galaxies as seen with the 100-m radio telescope at Effelsberg which has a half power beam width of $9.3^{\prime}$ at $21-\mathrm{cm}$ wavelength. As local $\mathrm{HI}$ is observed all over the sky our (ON-OFF) observing procedure produces the difference of the local emission between the source and the reference position at local velocities around zero $\mathrm{km} \mathrm{s}^{-1}$

galaxies have been further classified concerning probable membership by Karachentseva et al. (1985). Lo et al. (1986) conducted a deep IIIa-J survey of the M 81 group covering 36 fields using the 48 inch Palomar Schmidt. After visually searching the plates for low surface brightness objects, 137 candidate dwarf galaxies were detected of which 57 were judged to be group members. We composed a search list from the above named catalogs, which amounts to 136 candidate dwarf galaxies roughly in the range $65^{\circ} \leq$ Dec. $\leq 80^{\circ}$ and $7^{\mathrm{h}} 30^{\mathrm{m}} \leq$ R.A. $\leq 13^{\mathrm{h}}$. Most of these dwarfs have crude visual magnitudes of 17 to 19 mag (absolute magnitudes of -11 to -9 at the distance of the M 81 group), diameters around 1 arcmin or less, and classifications of type Im with a few E type or spheroidals (Börngen spheroidals were not detected in HI). A few galaxies are very close to brighter galaxies of this sample. This confusion could not be resolved as most probably the detections are due to the brighter galaxies.

\section{New Effelsberg HI observations}

Observations were performed in 1987/88 and 1991 using the $100 \mathrm{~m}$ radio telescope at Effelsberg which has a half power beam width of 9.3 arcmin for the $21 \mathrm{~cm}$ HI line. A FET receiver in the first run and a HEMT receiver in the latter run yielded a total system noise of $60 \mathrm{~K}$ and $30 \mathrm{~K}$ respectively. The total power mode was applied observing an on-source position followed by an empty reference field which was subtracted from the former in order to reduce instrumental effects. Pointing is believed to be about \pm 15 arcsec rms Some of the optical positions are not known to better than 1 arcmin. However, this is small compared to the telescope beam of 9 arcmin. Galaxies with 
optical velocities were observed with a velocity range of $1250 \mathrm{~km} \mathrm{~s}^{-1}$ (with a resolution of $6 \mathrm{~km} \mathrm{~s}^{-1}$ ). The radial velocity range of the M 81 group overlaps with velocities of local hydrogen. This "confusion" essentially could not be solved. Fainter signals adjacent to the range of velocities of local hydrogen were checked by both frequency modulation and rudimentary mapping. All such features which were suspected to be due to galaxies were found to be extended considerably larger compared to known extended haloes and to show velocity gradients different to what one would expect from a rotating galaxy. Therefore they were considered to be local emission.

We aimed for a detection limit of $10^{6}$ solar masses in $\mathrm{HI}$ at the distance of M 81. For comparison, the dwarf M $81 \mathrm{dwA}$ (Lo \& Sargent 1979) has an HI flux of $4.2 \mathrm{Jy}$ $\mathrm{km} \mathrm{s}^{-1}$ (line width of $22 \mathrm{~km} \mathrm{~s}^{-1}$ ) which corresponds to $7.210^{6}$ solar masses.

In order to identify background galaxies, a larger range of radial velocities had to be searched. The 1024 channel autocorrelator was split into four banks of 256 channels using a bandwidth of $6.25 \mathrm{MHz}$ each resulting in a resolution of $6 \mathrm{~km} \mathrm{~s}^{-1}$ and a velocity coverage from $-400 \mathrm{~km} \mathrm{~s}^{-1}$ to $4000 \mathrm{~km} \mathrm{~s}^{-1}$. Galaxies not detected in this velocity range were searched at higher radial velocities. In Fig. 2 we present the HI profiles of 65 of the 68 detected galaxies. All emission features at negative radial velocities fall into the range of local HI, only two cases are shown in Table 1 (objects 63 and 65), not shown are 211, K63, and 223. In Table 1 we present the observational data: the galaxy's name in Col. 1 (where a bare number refers to the catalog of Lo et al., a K to that of Karachentseva, and a BK to the catalog of Börngen et al.) is followed by the 1950 position used for the observation (Col.2), the galaxy type follows in Col. 3. H I data: the integrated HI flux (Col.4) in Jy $\mathrm{km} \mathrm{s}^{-1}$ is followed by the peak flux and the rms noise in Col. 5. The H I velocity (Col. 6) and the linewidths at $50 \%, 25 \%$, and $20 \%$ of the peak (Col. 7 ) are followed by comments (Col. 8). A colon (:) in Col. 7 marks a rather uncertain value.

Recently van Driel et al. (1997, A.A. submitted) searched for $\mathrm{HI}$ emission in 23 dwarfs in the area around M 81; they detected three background galaxies (UGC 4998, Kar 1N, and UGC 5658) and improved the existing upper limits.

The typical rms noise was in the range 0.003 to $0.01 \mathrm{~K}$; assuming line widths of 15 or $30 \mathrm{~km} \mathrm{~s}^{-1}$ (which are typical for small dwarf galaxies) and three times the rms noise this corresponds to an $\mathrm{HI}$ line integral of 0.1 to $0.6 \mathrm{Jy} \mathrm{km} \mathrm{s}{ }^{-1}$ or to 0.3 to $1.810^{6}$ solar masses of $\mathrm{HI}$ at the distance of the M 81 group (3.6 Mpc). This represents a detection limit much better than needed for the M 81dwA system. However this galaxy remains at the lower end of the HI fluxes of the detected objects. 68 galaxies (a detection rate of $49 \%$ ) were detected; most of which are background objects. Figure 2 shows the radial velocity distribution of these galaxies which is like that of a magnitude

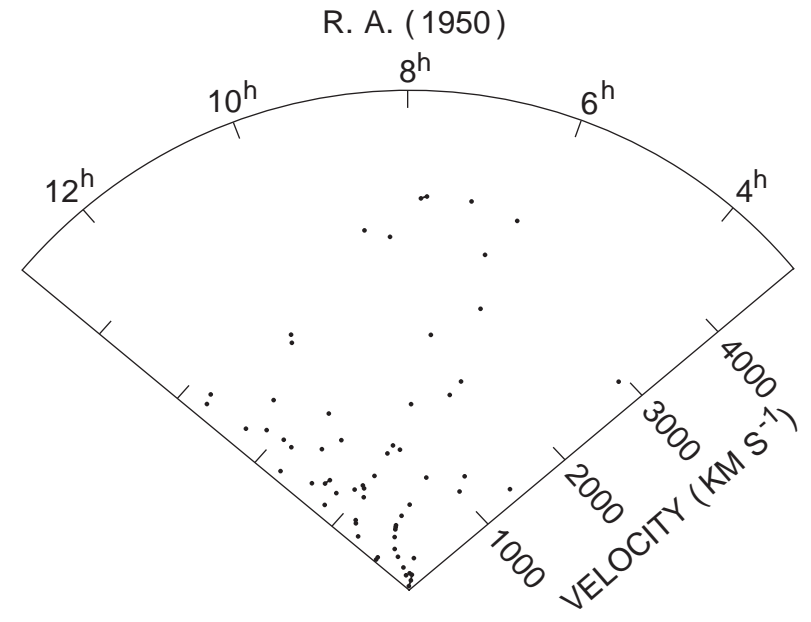

Fig. 2. The detected galaxies are plotted as a function of right ascension and radial velocity in this cone diagram. Only the very center $\left(v \leq 300 \mathrm{~km} \mathrm{~s}^{-1}\right)$ is the M 81 group

limited sample of galaxies rather than that of a group of galaxies at a certain distance.

\section{The M 81 group galaxies}

In Fig. 2 we present a cone diagram for the detected galaxies. Summed over the declination range 65 to 80 degrees the galaxies are displayed in R.A. versus radial velocity. Only the very center of this plot $\left(v \leq 300 \mathrm{~km} \mathrm{~s}^{-1}\right)$ represents the M 81 group. This cone diagram does not show a complete sample of galaxies, hence this diagram does not necessarily document the structure of the nearby universe. It is evident from this figure that all newly detected galaxies are far more distant than the M 81 group.

Galactic hydrogen in the foreground roughly covers a velocity range of -150 to $50 \mathrm{~km} \mathrm{~s}^{-1}$. About one third of the known M 81 group members have radial velocities in this interval. Therefore we probably miss a number of galaxies in this velocity interval due to confusion with the strong foreground emission of Galactic hydrogen.

\section{Conclusions}

This survey of dwarf galaxies in the M 81 group area revealed:

1) a detection rate of $49 \%$, most of which are background galaxies relative to the M 81 group, and

2) no new HI rich dwarfs in the M 81 group. With the detection limit in mind, this result is unexpected. HIobservations of the Centaurus and Sculptor groups (Côté 1995) yielded 9 new HI rich dwarf members around those groups with a detection limit of about $10^{7}$ solar masses.

While it is true that the velocity range covered by local neutral hydrogen prohibits detection of faint galaxies within this range, we would still expect at least a dozen or so dwarf galaxies to be detectable in the M 81 group if it 
had a luminosity function similar to that of the Centaurus and Sculptor groups. The undetected galaxies might be either low surface brightness galaxies in the background or dwarf ellipticals in the M 81 group itself.

In their study on dwarf galaxies Binggeli et al. (1990) concluded that "dwarf galaxies obey a morphology-density relation like the giants", and "dEs prefer strongly dense environments on all scales". Hence the different dwarf population of the Centaurus and Sculptor groups on one side and the M 81 group on the other side might just be documenting the large variety in the population of groups.

Acknowledgements. We are grateful to Wal Sargent for making available the catalog of M 81 group dwarf galaxy candidates prior to publication. This research has been supported, in part, by a NASA LTSARP grant (No. NAGW-3189) to EDS.

\section{References}

Binggeli B., Sandage A., Tammann G.A., 1985, AJ 90, 1681 Binggeli B., Tarenghi M., Sandage A., 1990, A\&A 228, 42

Börngen F., Karachentseva V.E., 1982, Astron. Nachr. 303, 189 Börngen F., Karachentseva V.E., Schmidt R., Richter G.M., Thanert W., 1982, Astron. Nachr. 303, 287

Bothun G.D., Impey C.D., Malin D.F., Mould J.R., 1987, AJ 93, 29

Côté S., 1995, Ph.D. thesis, Australia National University

van Driel W., Kraan-Korteweg R.C., Binggeli B., Huchtmeier W.K., 1977, A\&A (submitted)
Ferguson H.C., 1989, AJ 98, 367

Geller M.J., Huchra J.P., 1983, ApJS 52, 61

Huchtmeier W.K., Karachentsev I.D., Karachentseva V.E., 1997, A\&A (in press)

Huchtmeier W.K., Lercher G., Seeberger R., Saurer W., Weinberger R., 1995, A\&A 293, L33

Irwin M.J., Bunclark P.S., Bridgeland M.T., McMahon R.G., 1990, MNRAS 244, 16

Karachentseva V.E., 1968, Comm. Byurakan Obs. 39, 61

Karachentseva V.E., Karachentseva I.D., Börngen F., 1985, A\&AS 60, 213

Kraan-Korteweg R.C., Tammann G.A., 1979, Astron. Nachr. 300, 181 (KKT)

Kraan-Korteweg R.C., Loan A.J., Burton W.B., et al., 1994, Nat 372,77

Lo K.Y., Sargent W.L.W., 1979, ApJ 227, 756

Lo K.Y., Sargent W.L.W., Kowal, Smith, 1986, BAAS 18, 450

Madore B.F., Freedman W.L., Lee M.G., 1993, AJ 106, 2243

McCall M.L., Buta R.J., 1995, AJ 109, 2460

Shade D.J., Ferguson H.C., 1994, MNRAS 267, 889

Skillman E.D., Melnick J., Terlevich R., Moles M., 1988, A\&A 196, 31

Skillman E.D., Terlevich R., Melnick J., 1989a, MNRAS 240, 563

Skillman E.D., Kennicutt R.C., Hodge P.W., 1989b, ApJ 347, 875

Tully R.B., 1987, ApJ 321, 280

Tully R.B., 1988, Nearby Galaxies Catalog. Cambridge University Press

de Vaucouleurs G., 1975, in Galaxies and the Universe, Sandage A., Sandage M. \& Kristian J. (eds.). University of Chicago Press, Chicago, p. 557 
Table 1. Observed parameters

\begin{tabular}{|c|c|c|c|c|c|c|c|c|}
\hline $\begin{array}{r}\text { Galaxy } \\
\text { name } \\
\end{array}$ & \multicolumn{2}{|c|}{$\begin{array}{l}\text { position (1950.0) } \\
\text { R.A. Dec. }\end{array}$} & type & $\begin{array}{l}\text { HI flux } \\
{\left[\mathrm{Jy} \mathrm{km} \mathrm{s}^{-1}\right]}\end{array}$ & $\begin{array}{l}S_{\max } \\
{[\mathrm{mJy}]}\end{array}$ & $\begin{array}{c}\text { HI- } \\
\text { velocity }\end{array}$ & $\begin{array}{r}\text { HI-linewidths } \\
{\left[\mathrm{km} \mathrm{s}^{-1}\right]} \\
\end{array}$ & comment \\
\hline K37 & 032900 & 5755 & & 1.2 & $25 \pm 8$ & $1452 \pm 6$ & 889799 & \\
\hline K38 & 032900 & 6812 & & 3.5 & $33 \pm 8$ & $2969 \pm 5$ & 208330354 & \\
\hline $\mathrm{OC}$ & 051848 & 7316 & Irr & & \pm 10 & & & UGC 3297, multiple \\
\hline 201 & 051900 & 7624 & & & \pm 8 & & & diffuse \\
\hline OD & 052318 & 7225 & Irr & 11.7 & $224 \pm 8$ & $1089 \pm 1$ & 526971 & extremely diffuse \\
\hline UGC 3317 & 052730 & 7342 & Irr & 12.5 & $124 \pm 8$ & $1240 \pm 1$ & 110119121 & DDO 38 \\
\hline 202 & 055306 & 6833 & & & \pm 10 & & & \\
\hline 5 & 061106 & 7450 & $\operatorname{Irr}$ & & \pm 10 & & & smooth \\
\hline 7 & 062200 & 7048 & $\operatorname{Irr}$ & 2.3 & $50 \pm 11$ & $3860 \pm 3$ & 97105125 & \\
\hline $7 \mathrm{~A}$ & 062200 & 8254 & $\mathrm{~S} / \mathrm{Irr}$ & & \pm 11 & & & UGC 3461 \\
\hline 10 & 063836 & 8010 & $\mathrm{~S} / \mathrm{Irr}$ & & \pm 8 & & & \\
\hline 11 & 063842 & 6346 & Irr & 2.4 & $50 \pm 11$ & $2900 \pm 15$ & 677172 & \\
\hline 203 & 064054 & 8457 & & 6.3 & $66 \pm 8$ & $2134 \pm 5$ & 203210213 & diffuse \\
\hline UGC 3522 & 064100 & 8458 & $\mathrm{~S} / \mathrm{pec}$ & 9.0 & $75 \pm 7$ & $2132 \pm 5$ & 201212216 & \\
\hline 204 & 064536 & 6140 & & 4.2 & $100 \pm 6$ & $3450 \pm 2$ & 455557 & UGC 3545 \\
\hline 205 & 065236 & 6115 & & 7.1 & $75 \pm 8$ & $1970 \pm 2$ & 140142146 & \\
\hline 18 & 065912 & 6408 & & & \pm 17 & & & \\
\hline 20 & 070100 & 6601 & Irr & 2.8 & $32 \pm 8$ & $4174 \pm 5$ & 109148149 & \\
\hline K49 & 070242 & 7157 & & & \pm 5 & & & \\
\hline 22 & 070306 & 7830 & & & \pm 11 & & & UGC 3671 \\
\hline 23 & 070312 & 6522 & Irr & & \pm 8 & & & \\
\hline 25 & 070636 & 5920 & Irr & 4.9 & $33 \pm 7$ & $3968 \pm 15$ & 250: & \\
\hline 28 & 071036 & 6503 & Irr & & \pm 12 & & & \\
\hline 31 & 071648 & 6712 & Irr & & \pm 11 & & & \\
\hline 33 & 071930 & 6015 & Irr & & \pm 17 & & & \\
\hline 38 & 072318 & 6059 & Irr & & \pm 12 & & & \\
\hline 42 & 072548 & 6529 & Irr & & \pm 8 & & & \\
\hline 44 & 072936 & 7304 & Irr & 1.3 & $30 \pm 6$ & $2592 \pm 4$ & 789192 & \\
\hline 45 & 073054 & 6035 & Irr & 2.7 & $25 \pm 5$ & $300 \pm 10$ & 138171193 & \\
\hline 46 & 073442 & 5920 & $\mathrm{~S}$ & & \pm 8 & & & \\
\hline 50 & 074742 & 7336 & & 0.8 & $25 \pm 6$ & $3982 \pm 4$ & 99101122 & \\
\hline 207 & 075818 & 8617 & & 3.4 & $58 \pm 8$ & $1850 \pm 2$ & 587678 & \\
\hline UGC 4305 & 081406 & 7052 & Irr & 168.1 & $3104 \pm 20$ & $160 \pm 1$ & 536770 & DDO 50, HoII \\
\hline 63 & $08 \quad 14 \quad 12$ & 6305 & Irr & 11.7 & $590 \pm 8$ & $-180 \pm 1$ & 192830 & DDO 71 \\
\hline 64 & 081606 & 6551 & $\mathrm{~S} / \mathrm{Irr}$ & & \pm 8 & & & \\
\hline 65 & 081618 & 6618 & & 4.3 & $224 \pm 35$ & $-154 \pm 5$ & 424449 & \\
\hline K52 & 081842 & 7112 & Irr & 4.2 & $200 \pm 3$ & $112 \pm 5$ & 193036 & M81dwA \\
\hline UGC 4363 & 081918 & 7436 & $\mathrm{SBc}$ & 9.2 & $133 \pm 7$ & $3529 \pm 10$ & 6495105 & DDO 51 \\
\hline 68 & 081942 & 7431 & Irr & 3.7 & $83 \pm 11$ & $3450 \pm 5$ & 507080 & \\
\hline 69 & 082142 & 7900 & Irr & 2.1 & $75 \pm 8$ & $1399 \pm 6$ & 243234 & \\
\hline 70 & 082300 & 7226 & Irr & & \pm 12 & & & DDO 53 \\
\hline 73 & 083206 & 6958 & Irr & 13.0 & $380 \pm 8$ & $159 \pm 1$ & 324750 & UGC 4483 \\
\hline 75 & 083642 & 6953 & $\mathrm{~S}$ & & \pm 11 & & & \\
\hline UGC 4527 & 083800 & 7706 & Irr & 3.2 & $48 \pm 4$ & $720 \pm 3$ & 72140150 & \\
\hline 208 & 084054 & 7843 & & & \pm 17 & & & UGC 4563/4566 \\
\hline 79 & 084130 & 7843 & Irr & 3.9 & $44 \pm 8$ & $3634 \pm 5$ & 117128 158: & \\
\hline 82 & 084436 & 7338 & Irr & & \pm 22 & & & \\
\hline 84 & 084730 & 7827 & & 10.4 & $66 \pm 7$ & $1476 \pm 6$ & 187276293 & \\
\hline 210 & 085048 & 5840 & & 6.1 & $190 \pm 12$ & $-172 \pm 1$ & 333839 & \\
\hline & & & & 6.2 & $55 \pm 13$ & $3753 \pm 9$ & 238258263 & \\
\hline K53 & 085048 & 7212 & & & \pm 5 & & & diffuse \\
\hline 211 & 085318 & 6057 & & 10.4 & $280 \pm 8$ & $-175 \pm 1$ & 334748 & diffuse \\
\hline 212 & 085906 & 7906 & & & \pm 25 & & & very diffuse \\
\hline 213 & 090224 & 5933 & & & \pm 12 & & & \\
\hline 89 & 090900 & 6012 & & & \pm 11 & & & \\
\hline $90 \mathrm{~A}$ & 091730 & 8532 & $\mathrm{~S} / \mathrm{Irr}$ & 1.8 & $50 \pm 7$ & $1730 \pm 5$ & 284849 & \\
\hline K54 & 091736 & 755700 & & 2.4 & $120 \pm 15$ & $659 \pm 1$ & 152730 & \\
\hline U4998 & 092048 & 683700 & & & \pm 6 & & & \\
\hline $93 \mathrm{~A}$ & 092400 & 8618 & Irr & & \pm 8 & & & diffuse \\
\hline UGC 5139 & 093600 & 7135 & Irr & 38.7 & $1235 \pm 13$ & $143 \pm 1$ & 294044 & DDO 63, HoI, K57 \\
\hline
\end{tabular}


Table 1. continued

\begin{tabular}{|c|c|c|c|c|c|c|c|c|}
\hline $\begin{array}{r}\text { Galaxy } \\
\text { name }\end{array}$ & $\begin{array}{l}\text { position } \\
\text { R.A. D }\end{array}$ & $\begin{array}{l}(1950.0) \\
\text { Dec. }\end{array}$ & type & $\begin{array}{l}\text { HI flux } \\
{\left[\mathrm{Jy} \mathrm{km} \mathrm{s}^{-1}\right]}\end{array}$ & $\begin{array}{l}S_{\max } \\
{[\mathrm{mJy}]}\end{array}$ & $\begin{array}{c}\text { HI- } \\
\text { velocity }\end{array}$ & $\begin{array}{r}\text { HI-linewidths } \\
{\left[\mathrm{km} \mathrm{s}^{-1}\right]}\end{array}$ & comment \\
\hline 97 & 093624 & 5846 & $\mathrm{~S}$ & & \pm 12 & & & \\
\hline $1 \mathrm{~N}$ & 094100 & 693712 & & 4.2 & $85 \pm 6$ & $571 \pm 2$ & 536975 & \\
\hline 101 & 094318 & 7128 & Irr & & \pm 8 & & & diffuse \\
\hline BK2 & 094343 & 693024 & & & \pm 6 & & & \\
\hline 103 & 094606 & 6745 & & & \pm 12 & & & \\
\hline K59 & 094706 & 7219 & & 8.4 & $108 \pm 5$ & $1181 \pm 3$ & 140168171 & \\
\hline BK3 & 094942 & 691224 & & & \pm 11 & & & \\
\hline 215 & 095230 & 5831 & & & \pm 11 & & & \\
\hline 108 & 095630 & 6327 & & & \pm 12 & & & \\
\hline BK4 & 095956 & 682518 & & & \pm 11 & & & \\
\hline BK5 & 100044 & 682954 & & & \pm 8 & & & \\
\hline UGC5423 & 100125 & 703627 & & 3.4 & $76 \pm 5$ & $350 \pm 3$ & 475860 & \\
\hline K63 & 100136 & 6646 & & 4.2 & $133 \pm 3$ & $-126 \pm 5$ & 274350 & \\
\hline K64 & 100212 & 6803 & & & \pm 10 & & & \\
\hline UGC 5455 & 100454 & 7053 & Irr & 1.7 & $17 \pm 3$ & $428 \pm 8$ & 142182184 & K66 \\
\hline 117 & 101736 & 7121 & & & \pm 11 & & & \\
\hline UGC 5612 & 102006 & 7108 & SB & & \pm 6 & & & DDO 77 \\
\hline 120 & 102248 & 6754 & & 1.8 & $19 \pm 7$ & $2550 \pm 9$ & 210240255 & \\
\hline $\mathrm{U} 5658$ & 102354 & 7129 & & 1.2 & $37 \pm 7$ & $1126 \pm 1$ & & \\
\hline UGC 5666 & 102448 & 6840 & S & 184 & $2880 \pm 36$ & $57 \pm 2$ & 779195 & DDO 81 \\
\hline 123 & 102506 & 6325 & Irr & & \pm 11 & & & \\
\hline UGC 5671 & 102518 & 6704 & Irr & 4.6 & $68 \pm 5$ & $1136 \pm 2$ & 709194 & K69 \\
\hline $124 \mathrm{~A}$ & 102630 & 7430 & $\mathrm{~S}$ & 3.6 & $60 \pm 8$ & $2820 \pm 4$ & 120129132 & \\
\hline UGC 5688 & 102636 & 7019 & $\mathrm{SBm}$ & 13.0 & $266 \pm 15$ & $1920 \pm 2$ & 466176 & DDO 80 \\
\hline $126 \mathrm{~A}$ & 102730 & 7804 & Irr & 3.5 & $100 \pm 6$ & $1624 \pm 2$ & 384550 & UGC 5701 \\
\hline 126 & 102848 & 7053 & & & \pm 8 & & & DDO 82 \\
\hline $126 \mathrm{~B}$ & 103030 & 7924 & SB & 3.2 & $42 \pm 5$ & $2748 \pm 4$ & 102124127 & UGC 5728 126B \\
\hline BK6 & 103102 & $\begin{array}{lll}66 & 16 & 12\end{array}$ & & & \pm 6 & & & \\
\hline $127 \mathrm{~A}$ & 103900 & 5721 & Irr & & \pm 12 & & & diffuse \\
\hline UGC 5846 & 104112 & 6038 & Irr & 15.1 & $324 \pm 8$ & $1022 \pm 1$ & 485962 & DDO 86 \\
\hline 128 & 104124 & 7310 & $\mathrm{~S}$ & & \pm 8 & & & \\
\hline A $1045+65$ & 104530 & 6502 & & & \pm 6 & & & \\
\hline 129 & 104606 & 6500 & Irr & & \pm 12 & & & UGC 5932 \\
\hline K72 & 104618 & 6549 & Irr & 15.4 & $245 \pm 9$ & $340 \pm 1$ & 667679 & DDO 87 \\
\hline BK7 & 104707 & 652224 & & & \pm 6 & & & \\
\hline $\mathrm{A} 1047+65$ & 104712 & 6500 & & & \pm 6 & & & \\
\hline K73 & 104848 & 6956 & & 2.7 & $108 \pm 5$ & $-132 \pm 6$ & 273537 & \\
\hline UGC 5979 & 104912 & 6814 & Irr & 7.4 & $100 \pm 9$ & $1116 \pm 2$ & 90106109 & \\
\hline 134 & 104954 & 6948 & Irr & & \pm 12 & & & diffuse \\
\hline A $1050+65$ & 105030 & 6531 & & & \pm 7 & & & \\
\hline BK8 & 105106 & 652812 & & & \pm 7 & & & \\
\hline K74 & 105901 & 703206 & Irr & & \pm 8 & & & \\
\hline $137 \mathrm{~A}$ & 110412 & 6148 & Irr & 4.1 & $52 \pm 11$ & $1673 \pm 5$ & 768890 & \\
\hline 216 & 111448 & 6804 & & & \pm 17 & & & \\
\hline 138 & $\begin{array}{lll}11 & 19 & 12\end{array}$ & 6925 & $\mathrm{~S} / \mathrm{Irr}$ & & \pm 17 & & & UGC 6381 \\
\hline $138 \mathrm{~B}$ & 112436 & 7916 & pec & & \pm 4 & & & UGC 6456 \\
\hline 217 & 112442 & 7953 & & & \pm 8 & & & \\
\hline 218 & 113106 & 7638 & & & \pm 12 & & & \\
\hline 140 & 113130 & 7431 & & & \pm 17 & & & \\
\hline $140 \mathrm{~A}$ & 113236 & 5705 & & 1.7 & $42 \pm 12$ & $2317 \pm 2$ & 848689 & \\
\hline UGC 6682 & 114030 & 5923 & & 10.9 & $174 \pm 12$ & $1326 \pm 1$ & 688183 & \\
\hline 219 & 114612 & 5741 & & 4.1 & $66 \pm 11$ & $1200 \pm 4$ & 100112113 & \\
\hline UGC 6816 & 114806 & 5644 & & 13.8 & $137 \pm 9$ & $888 \pm 2$ & 116136139 & \\
\hline UGC 6912 & 115336 & 5829 & & 15.5 & $178 \pm 9$ & $1347 \pm 1$ & 91127130 & \\
\hline K84 & 115430 & 5633 & & & \pm 8 & & & \\
\hline $143 \mathrm{~A}$ & 115654 & 5722 & & 2.3 & $71 \pm 8$ & $849 \pm 2$ & 354446 & \\
\hline 143B & 115700 & 8526 & & 3.2 & $52 \pm 8$ & $1841 \pm 5$ & 100110118 & \\
\hline $143 \mathrm{C}$ & 115800 & 7908 & & 4.0 & $48 \pm 13$ & $1966 \pm 4$ & 91101104 & UGC 6996 \\
\hline 148 & 121018 & 6913 & & & \pm 12 & & & \\
\hline 150 & 121130 & 5950 & & & \pm 8 & & & \\
\hline $154 \mathrm{~A}$ & 121742 & 7527 & & 1.9 & $42 \pm 12$ & $1421 \pm 3$ & 869597 & \\
\hline
\end{tabular}


Table 1. continued

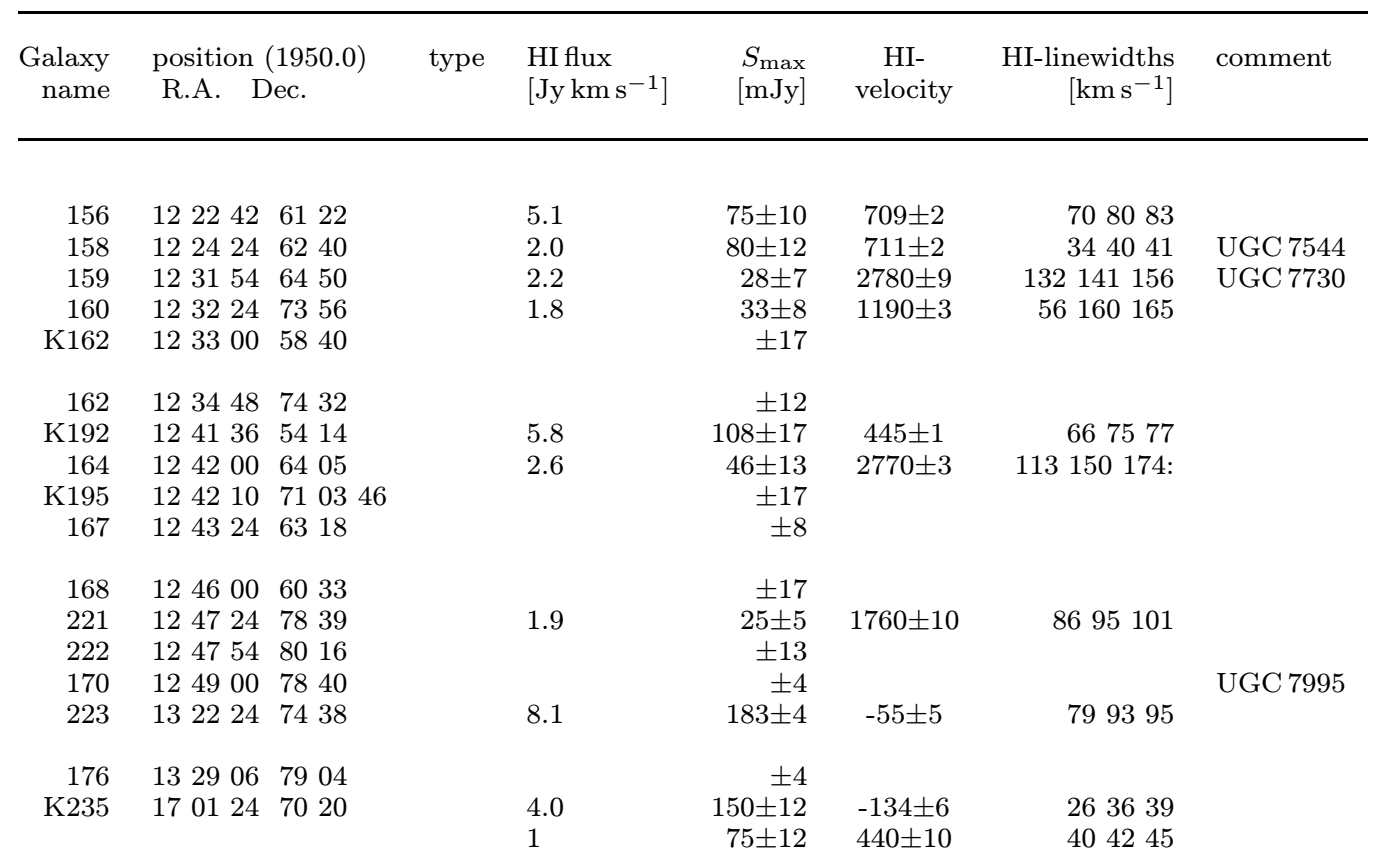

